\title{
Universiteit
}

Leiden

The Netherlands

\section{Experimental evidence for ice formation at room temperature} Jinesh, K.B.; Frenken, J.W.M.

\section{Citation}

Jinesh, K. B., \& Frenken, J. W. M. (2008). Experimental evidence for ice formation at room temperature. Physical Review Letters, 101(3), 036101. doi:10.1103/PhysRevLett.101.036101

Version: $\quad$ Not Applicable (or Unknown)

License: $\quad$ Leiden University Non-exclusive license

Downloaded from: https://hdl.handle.net/1887/64311

Note: To cite this publication please use the final published version (if applicable). 


\title{
Experimental Evidence for Ice Formation at Room Temperature
}

\author{
K. B. Jinesh ${ }^{* \dagger}$ and J. W. M. Frenken \\ Kamerlingh Onnes Laboratory, P.O. Box 9504, Leiden University, 2300 RA Leiden, The Netherlands
}

(Received 4 March 2008; published 15 July 2008)

\begin{abstract}
The behavior of water under extreme confinement and, in particular, the lubrication properties under such conditions are subjects of long-standing controversy. Using a dedicated, high-resolution friction force microscope, scanning a sharp tungsten tip over a graphite surface, we demonstrate that water nucleating between the tip and the surface due to capillary condensation rapidly transforms into crystalline ice at room temperature. At ultralow scan speeds and modest relative humidities, we observe that the tip exhibits stick-slip motion with a period of $0.38 \pm 0.03 \mathrm{~nm}$, very different from the graphite lattice. We interpret this as the consequence of the repeated sequence of shear-induced fracture and healing of the crystalline condensate. This phenomenon causes a significant increase of the friction force and introduces relaxation time scales of seconds for the rearrangements after shearing.
\end{abstract}

DOI: 10.1103/PhysRevLett.101.036101

PACS numbers: 68.35.Af, 61.20.- - , 68.37.Ps

When two objects slide past each other in ambient conditions, there are usually molecular-scale "third bodies" between them, such as water molecules or hydrocarbons, deposited from the ambient [1,2]. Many experiments exploring the atomic-scale origin of friction show that these trapped molecules can have considerable effects on the interaction between the sliding objects; some act like lubricants while others, on the contrary, increase friction [2]. Since the early 1990s there has been a thrust of research interest in this field, strongly fueled by molecular dynamics (MD) simulations that have identified various mechanisms by which adsorbates can affect friction and have quantified their influence on the forces between sliding objects [3-8]. The properties of lubricants at the molecular scale are still far from being understood completely and the experiments that have been reported so far have not yet reached molecular-scale sensitivity to the forces on individual confined molecules.

Simulations with ideal spherical lubricant molecules predict that when the lubricant is confined between two flat surfaces at a distance of more than 10 molecular diameters, it behaves simply as a Newtonian liquid $[4,6]$. But when the liquid film is squeezed down to a smaller thickness, it acquires a solidlike ordering of the molecules and exhibits a solidlike shear response $[5,9,10]$.

The behavior of confined water is intriguingly different from that of the organic liquids that have been studied the most $[11,12]$, some of these differences forming the subject of strong controversy [10-15]. When water is confined to a few monolayers, the translational entropy of the molecules is reduced so much that it becomes thermodynamically favorable for the confined liquid to solidify [12]. In the case of water, there are several factors that oppose the molecular ordering under confinement, such as the van der Waals interaction of the molecules with the confining surfaces and the nature of water to expand when freezing $[12,16]$. To solidify confined water into a highly oriented hydrogen-bonded network, these difficulties would have to be overcome in addition to the already required compensation of the large reduction in hydrogen-bond entropy with respect to the liquid phase. This has been proposed to make water stay liquid and retain a viscosity close to the value for bulk water, even under extreme confinement [11,12]. Nevertheless, recent simulations with water molecules between two flat mica sheets show water solidifying when confined to a thickness of one or two monolayers $(0.53 \mathrm{~nm})$, each molecule sharing hydrogen bonds with neighboring water molecules [14,15], resulting in the formation of monolayer and bilayer ice in the confined geometry. Recent experimental results show that the viscosity of confined water is 7 orders of magnitude higher than normal water at room temperature [17].

Here, we report direct experimental evidence for the formation of ice when water is confined between two objects at room temperature. We used a dedicated highresolution friction force microscope in which a tungsten tip is scanning over a highly oriented pyrolytic graphite (HOPG) surface. The special feature of our instrument is the cantilever - the Tribolever ${ }^{\circledR}$ - which is very flexible and thus sensitive in the two lateral directions and very stiff in the normal direction, making it ideal for friction measurements [18]. The cantilever used in the present experiment had a lateral spring constant of $6.1 \mathrm{~N} / \mathrm{m}$ and a normal spring constant of $30.5 \mathrm{~N} / \mathrm{m}$. The measurements were performed in a closed chamber, in which the relative humidity (RH) was controlled in the range of $1 \%$ to $50 \%$ by a continuous flow of mixtures of dry nitrogen and nitrogen humidified by blowing through distilled water. The scan range in all experiments was $3 \times 3 \mathrm{~nm}^{2}$ and the scan velocity was varied from $100 \mathrm{~nm} / \mathrm{s}$ down to $0.05 \mathrm{~nm} / \mathrm{s}$. A constant normal load of $3.7 \mathrm{nN}$ was used throughout the experiments.

Figures 1(a)-1(c), show three typical force loops obtained at a low RH of $1 \%$ and at three different tip velocities. At a velocity of $30 \mathrm{~nm} / \mathrm{s}$ [Fig. 1(c)], the forward and reverse curves exhibit the familiar sawtooth signature of 


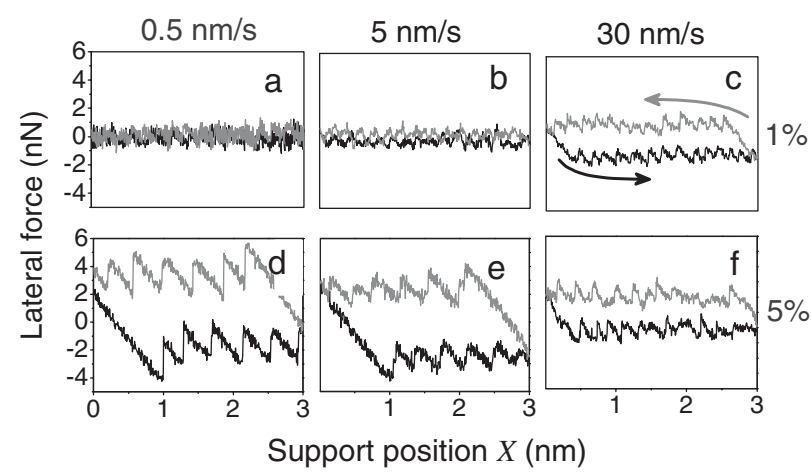

FIG. 1. Measurements of the lateral force between a tungsten tip and a graphite surface, scanning back and forth over a distance of $3 \mathrm{~nm}$ at three different velocities $(v)$ and two relative humidities (RH): (a) at $\mathrm{RH}=1 \%$ and $v=0.5 \mathrm{~nm} / \mathrm{s}$, stochastic thermal jumps of the tip between the neighboring wells in the tip-substrate potential lead to very low friction; (b) at $\mathrm{RH}=1 \%$ and $v=5 \mathrm{~nm} / \mathrm{s}$, graphite lattice appears over thermal noise, (c) at $\mathrm{RH}=1 \%$ and $v=30 \mathrm{~nm} / \mathrm{s}$, the tip performs stick-slip motion with the $0.25 \mathrm{~nm}$ period of the graphite lattice; (d) at $\mathrm{RH}=5 \%$ and $v=0.5 \mathrm{~nm} / \mathrm{s}$, the tip experiences strong friction and undergoes pronounced stick-slip motion with a period of approximately $0.4 \mathrm{~nm}$; (e) at $\mathrm{RH}=5 \%$ and $v=5 \mathrm{~nm} / \mathrm{s}$, the slip events become more noisy, (f) at $\mathrm{RH}=5 \%$ and $v=$ $30 \mathrm{~nm} / \mathrm{s}$ velocity, the friction force is lower and the graphitic lattice reappears.

atomic stick-slip motion with the $0.25 \mathrm{~nm}$ period of the graphite lattice. When the velocity is reduced, the average lateral force (friction force) becomes smaller, which we ascribe to thermally assisted, spontaneous forward (and reverse) jumps of the tip between neighboring potential wells of the tip-graphite interaction $[19,20]$. At a velocity of $0.5 \mathrm{~nm} / \mathrm{s}$ [Fig. 1(a)], the friction force is as small as $200 \mathrm{pN}$. At the modest RH of 5\% the behavior is strikingly different. Although the force loop at $30 \mathrm{~nm} / \mathrm{s}$ [Fig. 1(f)] is similar to (albeit somewhat noisier than) that at $\mathrm{RH}=1 \%$ [Fig. 1(c)], the friction force at $0.5 \mathrm{~nm} / \mathrm{s}$ is significantly higher [Fig. 1(d)], rather than being vanishingly small. How the friction force varies with velocity is plotted in Fig. 2 for the two humidities. The behavior becomes increasingly different as the velocity is lowered below $10 \mathrm{~nm} / \mathrm{s}$. Measurements over a range of humidities show similarly high friction at low velocities for intermediate RH values, between 5\% and 35\%.

Surprising in all low-velocity $(v<3 \mathrm{~nm} / \mathrm{s})$ friction loops at intermediate humidities $(\mathrm{RH}=5 \%-35 \%)$ is the pronounced stick-slip character of the tip motion with a completely new period of approximately $0.4 \mathrm{~nm}$ [Fig. 1(d)]. This period does not depend on the precise conditions, such as the scan velocity and the humidity. Figure 3 shows the distribution of stick-slip distances, measured in the range of $\mathrm{RH}$ from $5 \%$ to $35 \%$ and for velocities ranging from 0.5 to $3 \mathrm{~nm} / \mathrm{s}$, from which we derive an average stick-slip distance of $0.38 \pm 0.03 \mathrm{~nm}$. The unusually low scan velocities used here require ex-

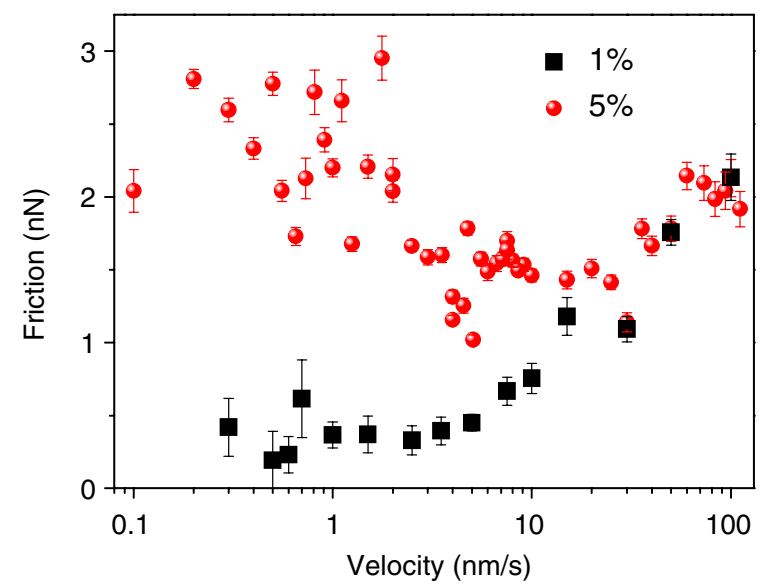

FIG. 2 (color online). Velocity dependence of the friction force (average lateral force) at two different humidities. At $\mathrm{RH}=1 \%$, friction follows a sublinear velocity dependence due to the thermally activated jumps of the tip in the tip-graphite interaction potential [19]. At $\mathrm{RH}=5 \%$, the friction force is unexpectedly high at velocities below $10 \mathrm{~nm} / \mathrm{s}$.

treme stability of the friction force microscope, the lowest scan velocity corresponding to a scan time of 2 minutes per force loop. From the comparison between the forward and reverse parts of the low-velocity loops [e.g., Fig. 1(d)] we know that our lateral drift speed has been below $0.01 \mathrm{~nm} / \mathrm{s}$, which is low enough not to influence the force loops noticeably.

At even higher humidities, e.g., 50\%, the low-velocity friction remains high, but the stick-slip variations are ab-

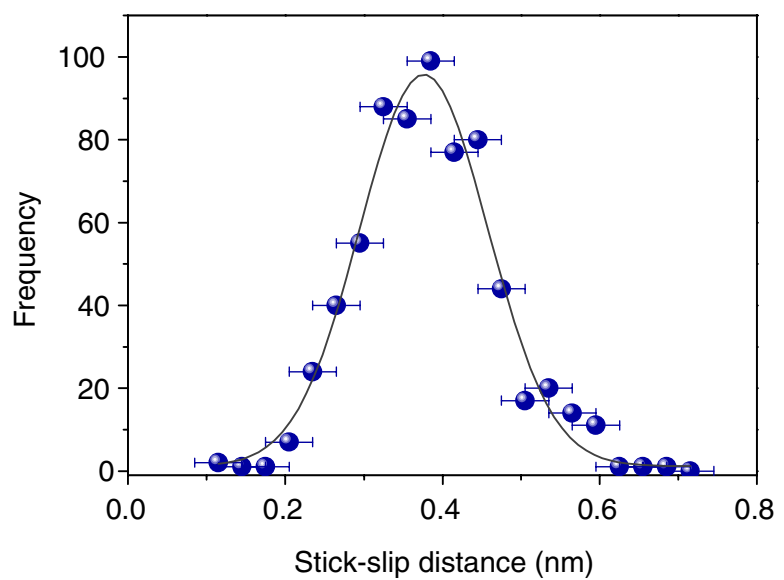

FIG. 3 (color online). Distribution of stick-slip distances observed at low velocities $(0.1 \mathrm{~nm} / \mathrm{s}$ to $3 \mathrm{~nm} / \mathrm{s})$ and modest $\mathrm{RH}$ values (5\% to $33 \%$ ). The gray curve is a Gaussian fit to the data, from which we derive an average stick-slip distance of $0.38 \pm$ $0.03 \mathrm{~nm}$. (The error-bar includes the systematic error in the measurements and the calibration error of the instrument). This value lies well within the range of lattice constants of 0.27 to $0.45 \mathrm{~nm}$ corresponding the known structures of crystalline ice. 
sent. These high-RH observations have been reported before [2]. They have been interpreted as the result of capillary condensation of water between the tip and the graphite surface. This condensate was shown to respond elastically to shear forces up to an estimated yield stress of $90 \mathrm{MPa}$, similar to that of bulk ice. The typical time scale for rearrangements of the condensate after plastic deformation was shown to be in the order of a second. In the present experiments we find that at a relative humidity of 5\% the long-period stick-slip motion persists up to a velocity of $3 \mathrm{~nm} / \mathrm{s}$; at higher velocities the loop gradually changes from pronounced stick-slip to a wavy shape with the same long period, as shown in Fig. 1(e). At even higher velocities, above $9 \mathrm{~nm} / \mathrm{s}$, the graphite lattice period emerges. At a humidity of $33 \%$, we observed that the pronounced longperiod stick-slip motion extends only to velocities of $\sim 1 \mathrm{~nm} / \mathrm{s}$ followed by the wavy pattern. At higher velocities, we do not observe any sign of the graphite lattice at this humidity.

Since the long-period stick-slip motion reported here appears only at nonzero relative humidities, we conclude that also this effect is a consequence of capillary condensation between the tip and the graphite surface. However, we may expect that at the more modest humidities than those in Ref. [2], the thickness of the capillary condensate will be smaller [21], leading to an even stronger tendency for the water molecules to order.

Unfortunately, we have been able to measure the repeat distance of $0.38 \pm 0.03 \mathrm{~nm}$ only along a single direction and due to the required, extremely slow scan speeds, we have no reliable information about the structure in the direction perpendicular to the fast scan direction. Nevertheless, we speculate that this distance is associated with the lattice of a regular, icelike capillary film. There is a variety of known ice structures, including the most common hexagonal lattice with periods of $0.27 \mathrm{~nm}$ and $0.45 \mathrm{~nm}$ and the metastable crystalline phase of ice XII, where the unit cell is constituted by lattice vectors with $a=0.83 \mathrm{~nm}$ and $c=0.402 \mathrm{~nm}$ [22]. Modifications to these lattice constants can result from the interaction with the surface on which the ice monolayers form [23]. There are two scenarios that would provide friction loops with stick-slip behavior with the ice lattice. In the first, the tungsten tip simply scans over the surface of a thin, crystalline layer of ice on the graphite substrate. In the second, the ice is firmly attached, both to the tip and to the graphite, and the ice is repeatedly forced to break (shear melt) and reattach (resolidify) after a translation over a single lattice period. The high shear force observed in our measurements strongly suggests that it is the latter scenario that applies. In principle, both the tip and the surface can induce ordering in the confined liquid independently, as many investigations on the interface of liquids on atomically smooth crystals reveal $[2,14,24-26]$. Recent MD simulation studies report that confinement to less than a nanometer width can promote solidification of water in hydrophobic pores above its freezing temperature [27].

To obtain further evidence for the regular (ice) structure of the capillary condensate, we measured the dependence of the friction force on the normal force (load) at a relative humidity of $25 \%$. The result is shown in Fig. 4. We recognize various plateaus in this plot, which are indicated by rectangles. We associate the steps between these plateaus with the squeeze out of subsequent water (ice) monolayers from the confined geometry when the normal load is increased. From this, we see that the condensate is also ordered in the normal direction and we estimate that there are at least 3 monolayers of ice confined between the tip and the surface at this relative humidity.

The fact that the long-period stick-slip motion is exclusively observed at ultralow velocities and that it becomes more pronounced the lower the velocity is, indicates that the time scale for the removal of the shear-deformationinduced damage is on the order of $1 \mathrm{~s}$, a time scale very reminiscent of that found for shape rearrangements of the condensate at higher humidities in Ref. [2]. When the humidity is increased, the ice film should become somewhat thicker [21]. We speculate that this reduces the crystalline quality of the film and increases its time scale for ordering, thus explaining that the maximum velocity at which the ice lattice is observed is lower at higher humidities. Also the velocity weakening observed in Fig. 2 at $\mathrm{RH}=5 \%$ below $10 \mathrm{~nm} / \mathrm{s}$ supports the damage removal scenario. As the velocity is increased, the film is given less time to heal out the shear-induced damage, which reduces not only the stick-slip amplitude, but also the shear strength of the film.

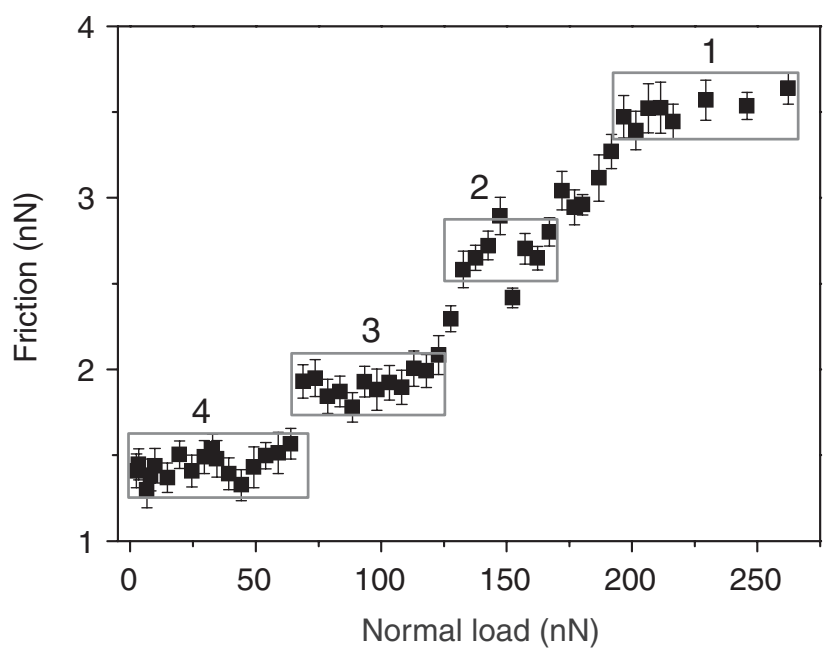

FIG. 4. Dependence of friction on the normal force, measured at $\mathrm{RH}=25 \%$ at a constant velocity of $30 \mathrm{~nm} / \mathrm{s}$. The gray boxes indicate the presence of force plateaus, which forms clear evidence of water (ice) being squeezed out of the contact layer by layer when the normal load is increased. 


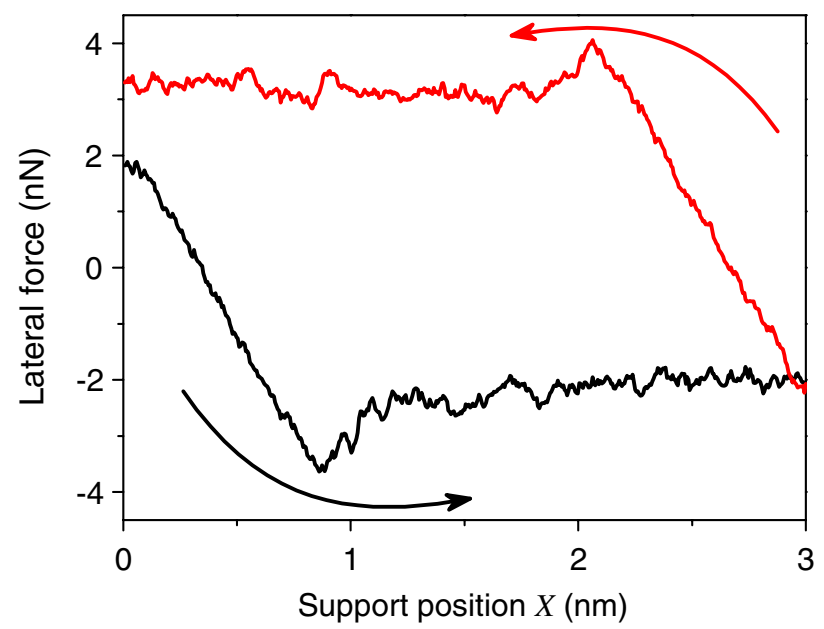

FIG. 5 (color online). A typical lateral force loop at $\mathrm{RH}=$ $33 \%$ at a velocity of $30 \mathrm{~nm} / \mathrm{s}$. After the turning points, the lateral force at this relatively high velocity passes through an overshoot, visible as the "static peaks" in the left-to-right and right-to-left curves (lower left and upper right, respectively), each followed by a decay to a substantially lower kinetic friction force. The absence of graphite lattice features in the force loop shows that the tip is not in direct contact with the graphite substrate, suggesting the presence of intervening water. The fully elastic behavior around the turning points indicates that this water still behaves like a solid, while the absence of icelike lattice features at this high velocity implies that the ice is significantly disordered. We interpret the static peak as the signature that the condensate somewhat recovers in crystal quality and therefore in yield strength at the turning pointsthe residence time was $0.3 \mathrm{~s}$ at the left turning point and $0.03 \mathrm{~s}$ at the right.

An interesting consequence of this behavior is the natural introduction of static friction. When a scan is briefly interrupted, the healing process has more time and is more complete, resulting in a high initial lateral force peak before the system resumes its steady-state stick-slip motion. Figure 5 illustrates that we indeed observe such a transient peak at higher velocities, where the long-period stick-slip amplitude has reduced to nearly zero. This peak, which overshoots the dynamic friction level by as much as $40 \%$, is seen both at the beginning of each trace from left to right (at $X=0.8 \mathrm{~nm}$ in Fig. 5) and at the beginning of each return trace (at $X=2.1 \mathrm{~nm}$ ).

Together, the four observations presented in this Letter, (i) the low-velocity increase in friction, (ii) the new lattice period in the stick-slip motion, different from that of graphite but similar to that of various ice types, (iii) the layering derived from the normal force dependence of the friction force, and (iv) the extremely slow build-up of static friction, form strong evidence that a monocrystalline water condensate is formed between a tungsten tip and a graphite surface at room temperature. These findings put the controversial subject of water under nanoscale confinement into a new light.

*Present address: NXP Semiconductors, High Tech Campus 4, 5656 AE, Eindhoven, The Netherlands.

Corresponding author. k.b.jinesh@nxp.com

[1] M. Binggeli and C. M. Mate, Appl. Phys. Lett. 65, 415 (1994).

[2] K. B. Jinesh and J. W. M. Frenken, Phys. Rev. Lett. 96, 166103 (2006).

[3] G. S. Blackman, C. M. Mate, and M. R. Philpott, Phys. Rev. Lett. 65, 2270 (1990).

[4] G. He, M.H. Müser, and M. O. Robbins, Science 284, 1650 (1999).

[5] J. N. Israelachvili, P. M. McGuiggan, and A. M. Homola, Science 240, 189 (1988).

[6] M. L. Gee, P. M. McGuiggan, and J. N. Israelachvili, J. Chem. Phys. 93, 1895 (1990).

[7] J. H. Cushman, Nature (London) 347, 227 (1990).

[8] J. Klein and E. Kumacheva, J. Chem. Phys. 108, 6996 (1998).

[9] B. Bhushan, J. N. Israelachvili, and U. Landman, Nature (London) 374, 607 (1995).

[10] A. L. Demirel and S. Granick, Phys. Rev. Lett. 77, 2261 (1996).

[11] U. Raviv, S. Perkin, P. Laurat, and J. Klein, Langmuir 20, 5322 (2004).

[12] U. Raviv, P. Laurat, and J. Klein, Nature (London) 413, 51 (2001).

[13] S. Granick, Science 253, 1374 (1991).

[14] Y.S. Chu et al., Phys. Rev. Lett. 86, 3364 (2001).

[15] R. Zangi and A.E. Mark, Phys. Rev. Lett. 91, 025502 (2003).

[16] E. A. Jagla, Phys. Rev. Lett. 88, 245504 (2002).

[17] R. C. Major et al., Phys. Rev. Lett. 96, 177803 (2006).

[18] M. Dienwiebel et al., Rev. Sci. Instrum. 76, 043704 (2005).

[19] S. Yu Krylov et al., Phys. Rev. E 71, 065101 (2005).

[20] E. Gnecco et al., Phys. Rev. Lett. 84, 1172 (2000).

[21] J. N. Israelachvili, Intermolecular and Surface Forces. (Academic, London, 2002), 2nd ed.

[22] C. Lobban, J. L. Finney, and W. F. Khus, Nature (London) 391, 268 (1998).

[23] M. F. Reedijk et al., Phys. Rev. Lett. 90, 066103 (2003).

[24] P. B. Miranda, L. Xu, Y. R. Shen, and M. Salmeron, Phys. Rev. Lett. 81, 5876 (1998).

[25] A. Gil et al., Langmuir 16, 5086 (2000).

[26] K. Koga, H. Tanaka, and X. C. Zeng, Nature (London) 408, 564 (2000).

[27] L. Cheng et al., Phys. Rev. Lett. 87, 156103 (2001). 